\title{
Scheduling an amateur cricket league over a 9-year period
}

\begin{abstract}
This paper describes a scheduling exercise carried out for the Minor Counties' Cricket Association (MCCA), which runs an amateur league based across England. The MCCA League consists of two wholly separate divisions of ten teams each, with each team playing three home matches and three away matches against teams in their own division each year, with opponents rotating between years; effectively this was scheduled as a double round robin over a three-year period.

Originally the schedules had been repeated on a three-year cycle. However, problems of fairness and balance between years arose, and the MCCA therefore decided they needed to commission the creation of a nine-year schedule - a sextuple round-robin - which would address these equity issues and others. These issues were formulated as soft constraints, some of which related to a nine-year period, and a schedule was successfully produced using a form of Simulated Annealing, operating over a variety of neighbourhoods. The new nine-year schedule is currently in operation.
\end{abstract}

Keywords: Sports, cricket, scheduling, simulated annealing, heuristics

\section{Sports scheduling}

All sporting competitions need to be scheduled. At the most basic level, participants need to know when and where to turn up. All schedules are subject to hard constraints (for example, participants can usually take part in no more than one contest at any given time, though there are exceptions such as simultaneous chess tournaments) and often also soft constraints (regarding features that are not infeasible but are certainly not desirable). Even if all constraints can be obeyed, some schedules will be better than others (though what "better" means is not always easy to define).

Thus, to an Operational Research (OR) analyst, sports scheduling (sometimes referred to as "sports timetabling") falls neatly into the category of optimisation problems, and it is a field that has interested both OR theoreticians and those seeking implementable solutions to real problems.

Many papers have been published in the academic literature regarding the practical implementation of sports scheduling, covering a wide variety of sports, including cricket (e.g. Willis \& Terrill (1994), Wright $(1994,2005))$. Other sports featured include Australian football (Kyngäs et al., 2016), football (Recalde et al., 2013), ice hockey (Nurmi et al., 2014), basketball (Westphal, 2014), rugby union (Johnston \& Wright, 2014), volleyball (Meng et al., 2014), athletics (Lin \& Yang, 2010), tennis (della Croce et al., 1999), softball (Saur et al., 2012) and others.

A very common type of sporting competition structure where high-quality scheduling can be both important and difficult is the league system, whereby teams are paired against one another over a pre-defined set of time periods. This can take a variety of formats, such as single round-robin, where every team opposes every other team exactly once, double round-robin, where every team opposes every other team twice, once at the home venue of each team, and other more complex systems. For example, Nurmi et al. (2014) scheduled an ice hockey tournament with a triple round robin structure; Kyngäs et al. (2016) scheduled an Australian Football tournament with a structure which was part way between a single and a double round robin, such that some pairs of teams played each other twice while other pairs played each other only once; while Johnston \& Wright (2014) scheduled a Rugby Union tournament which involved a single round robin within divisions plus a selection of interdivisional matches. 
While most published implementations involve professional sport or "almost professional" sport (e.g. in the American college system), there are also papers which report on implementations involving truly amateur sports competitions, for which the main issues are often different. For example Capua et al. (2012) needed to take account of age-related restrictions and the sharing of coaches when scheduling the training of youth football teams; Goossens \& Spieksma (2011) scheduled an indoor football league where a minimum number of days between matches was essential because of participants' other commitments; and Schönberger et al. (2004)'s work for a table tennis league needed to take account of limited availabilities of both participants and facilities. None of these considerations would normally apply to professional sporting competitions.

Every sports scheduling problem has its own characteristics and a wide variety of solution approaches has been used, including (meta)heuristic and exact mathematical methods. For example, Saur et al. (2012) and Recalde et al. (2013) used mathematical programming approaches; Lin \& Yang (2010) used a Genetic Algorithm; Schönberger et al. (2004) used a Memetic Algorithm; Willis \& Terrill (1994) used Simulated Annealing; Wright (1994) used a variant of Tabu Search; and Kyngäs et al. (2016) used PEAST, a sophisticated variant of local search. Indeed, almost every implementation is slightly different from every other one; even when a similar method has been used, the exact details are different, often because of differences between the individual features of the problems addressed.

Kendall et al. (2010) \& Trick (2011) have written survey papers which highlight a substantial amount of academic literature on the subject of sports scheduling. In addition, Wright (2009), which represents an overview of OR in Sports, devotes a considerable section to scheduling, and Wright (2010) summarises the most important OR and Management Science work relating to cricket, the sport considered in this paper.

\section{Minor Counties Cricket}

The Minor Counties Cricket Association (MCCA) runs a league competition known as the Minor Counties Cricket Championship. "Minor Counties", in cricketing terms, are counties (as defined before the 1974 restructuring of counties within the UK) without a professional men's cricket team, spread right across England and also encroaching into Wales. For some time the structure has been for minor counties to be divided into two geographically-based leagues (the Eastern and Western Divisions) for the purposes of this particular competition (there are other competitions, but these are not of concern in this paper). The geographical basis for these divisions is only approximate - for example, Cumberland and Staffordshire in the Eastern Division are in fact considerably to the West of Oxfordshire and Berkshire in the Western Division.

This paper concerns an issue raised by counties in the Eastern Division. See Figure 1 for a map showing these counties; as can be seen, some of the distances involved are not small; at the extreme, according to the Automobile Association (www.theaa.com), a car journey from Maryport in Cumberland to Ipswich in Suffolk would be expected to take 6 hours and 41 minutes. 


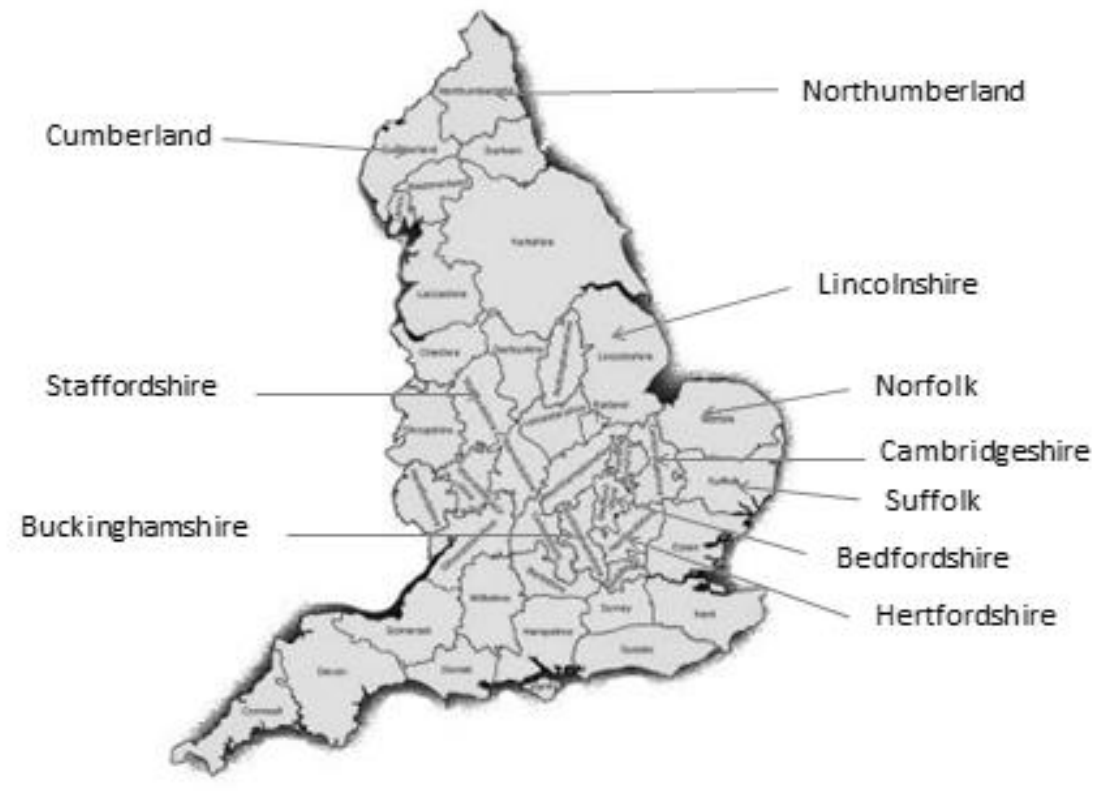

Figure 1 - Eastern Division Counties

Within each Division, every county team plays three home matches and three away matches each year against other teams in its Division, and the Divisional champions are determined according to the results of these matches every year. Matches are played over three days - usually Sunday, Monday and Tuesday - and generally occur at two-weekly intervals between early June and mid-August.

However, this is clearly an unbalanced schedule, falling well short of a round robin; in any given year, a team will avoid having to play against three other teams in its Division, potentially giving the team an unfair (dis)advantage. This is rectified over a three-year period by ensuring that every team plays at home exactly once and away exactly once against every other team in its Division, with the home and away match for every pairing always taking place in different years. This is thus equivalent to a double round robin structure over three years.

Until 2015, the manually constructed schedules were repeated on a three-year cycle - thus the schedule for 2014 was the same as that for 2011, the 2013 the same as that for 2010, etc. However, some counties in the Eastern Division were not happy with this arrangement because of a specific feature introduced to accommodate one county, as detailed below, and thus requested a change to the system as from 2015. These issues did not arise in the Western Division and therefore there was no impetus for change in that Division.

\section{Issues within the Eastern Division}

The Norfolk Cricket Festival is a tradition which goes back for many decades, which developed from an original Norfolk Cricket Week dating from 1881 (see

http://www.norfolkcountycricketclub.co.uk/about-the-club/club-history). The Festival now involves Norfolk playing all their home matches in the Minor Counties Cricket Championship in three consecutive weeks in July and August, thus requiring an exception to the standard scheduling structure of a round every two weeks.

Tradition is very important in cricket, especially at the amateur level, and therefore the MCCA has accommodated this Festival every year by initially scheduling home matches for Norfolk in Round 4, Round 5 and either Round 2 or Round 3 , but then rescheduling the latter match to a slot between 
Rounds 4 and 5 (call it Round 4a). Thus, for example, Norfolk's home matches during 2014 were against Cumberland between July $20^{\text {th }}$ and July $22^{\text {nd }}$; against Bedfordshire between July $27^{\text {th }}$ and July $29^{\text {th }}$; and against Suffolk between August $3^{\text {rd }}$ and August $5^{\text {th }}$.

However, while this arrangement satisfied Norfolk, some other counties were not so happy. In particular, those counties that were scheduled to play in Norfolk in Round 4a every three years (Cambridgeshire, Buckinghamshire and Bedfordshire) often found that some of their players were unwilling or unable to play in all of Rounds $4,4 a$ and 5 . These are not professional players; to take two days off work (and also time away from domestic duties for away matches) three weeks in a row may not always be possible or popular. Moreover, it was possible that family holidays would take players away from two or even three consecutive matches when they were scheduled so close together.

In addition, the system of repeating the schedule every three years had led to a lack of variety which some counties were not happy with, especially because of some imbalances of home matches even within the three-year schedule. For example:

- Buckinghamshire were always at home in Rounds 2 and 4;

- Lincolnshire were always at home in Round 1;

- Cambridgeshire were always at home in Round 3;

- Cumberland were always at home in Round 6;

- Suffolk only ever had one home match during the summer holidays (Rounds 4, 5 and 6).

These features had arrived by chance, not because of any request from the counties.

Another concern derived from the fact that some counties use more than one ground, and often tend to use them in the same order because of patterns of availability (they all have to borrow grounds from local clubs). The repeating nature of the schedule thus determined that some opposing counties might visit a particular ground every three years, but that other counties in the division would never play there. Both home teams and away teams reported that they would prefer more variety.

The MCCA therefore decided that, while they wished to keep the pattern of home and away opponents for each year the same as in the three-year schedule, they needed new schedules for these matches for the years from 2015 onwards. One possibility could have been to come up with a new schedule every three years, but their preferred option was to create a nine-year schedule: this can be regarded as a sextuple round robin, with some constraints cutting across not only all nine of these years but also the three preceding years, as described below. The intention was that this nine-year schedule would then repeat ad infinitum, or at least as long as the membership of the Eastern Division and the format of the competition remain as they are now. As far as can be ascertained, this is the longest sports scheduling horizon in practical use.

\section{The scheduling problem}

The matches (ordered pairings of teams) were thus predetermined for every year; the problem was to assign a Round ( 1 to 6 plus 4a) to every match to be played in every year from 2015 to 2023 inclusive. The matches within the years 2012 to 2014 were also included in the problem because they affected some of the soft constraints (see below), but they were constrained to be assigned to the Rounds in which they had actually taken place. The only hard constraint was that, within any given year, no team could play two matches within the same round. Other considerations were modelled by a variety of soft constraints with widely differing penalty costs, as follows.

The Match Absence cost was 10000 for each match not scheduled for any year from 2015 to 2023. The high cost reflects the fact that, although the system treated this as a soft constraint rather than a hard one, it was clear that no solution would be acceptable if there were any Match Absence cost. 
The Stated Preference costs were 1000 for not providing a home match in a requested Round, or providing a home match when a team had requested not to have one, for any year from 2015 to 2023; or 500 for not acceding to any other request. Here the requests of the former type were for Norfolk to have home matches in Rounds 4, 4a and 5 every year; for no other team to have a home match in Round $4 a$ in any year; and for Bedfordshire to have a home match in Round 4 every year. The only request of the latter, more complex type, was for Northumberland not to have a home match in more than one of the first three Rounds, because of the limited availability of their preferred ground, Jesmond, during June and early July (the 2014 schedule had given them two home matches during this period, which had both had to be played at other grounds within Northumberland).

The Number of Matches cost applied when any Round, in any year from 2015 to 2023, did not have the required number of matches scheduled in it ( 5 for Rounds $1,4,5$ and $6 ; 4$ or 5 for Rounds 2 and 3; 1 for Round 4a; and 0 for the dummy Round 7 (see later)). The cost was 50 times the square of the difference between the number of matches and the required number. The square of the difference was used because it was perceived that one difference of two was worse than two differences of one.

The Home/Away Balance cost concerned the way that home and away matches were spread between the Rounds within each year; this is a common criterion used in scheduling implementations. Within each year from 2015 to 2023, for every team except Norfolk, the following costs applied:

- 250 for a team at home in rounds $\{1,2,3\}$ or $\{4,5,6\}$;

- 250 for a team away in rounds $\{1,2,3\}$ or $\{4,5,6\}$ or $\{4 a, 5,6\}$;

- 50 for a team at home in rounds $\{2,3,4\}$ or $\{3,4,5\}$;

- 50 for a team away in rounds $\{2,3,4\}$ or $\{3,4,5\}$ or $\{3,4,4 a\}$ or $\{4,4 a, 5\}$;

- 25 for a team at home in rounds $\{1,2\}$ or $\{5,6\}$;

- 25 for a team away in rounds $\{1,2\}$ or $\{5,6\}$;

- 5 for a team at home in rounds $\{2,3\}$ or $\{3,4\}$ or $\{4,5\}$;

- 5 for a team away in rounds $\{2,3\}$ or $\{3,4\}$ or $\{4,5\}$ or $\{4,4 a\}$ or $\{4 a, 5\}$.

The reason for the asymmetry between home and away costs was that the stated preference costs already penalised heavily any instance of a team other than Norfolk being at home in Round 4a.

The Fixture Spread cost concerned the Rounds in which specific matches (i.e. ordered parings of \{home, away\} teams) were scheduled. Any specific pairing only occurs every three years, and thus during four years during the period 2012-2023. If a match occurred in the same Round twice the cost was 20 ( 400 for Round 4a) if the incidences were three years apart; 8 (again 400 for round $4 a$ ) if the incidences were six years apart; and 2 if they were nine years apart. The higher costs for round $4 a$ reflect the fact that repetition in Round 4a was the main impetus for this work to be carried out.

The Home Round Spread cost concerned the spread over the years of rounds in which a team other than Norfolk was to be at home. If a team was at home in the same slot for three consecutive years, there was a cost of 3 . If a team was at home in a particular slot more than six times or less than three times in any set of nine consecutive years within the period 2012-2023, the cost was 1 times the square of the discrepancy, i.e. 9 for zero or nine times, 4 for one or 8 times, 1 for two or 7 times. Again, the square of the discrepancy was used because it was perceived that one discrepancy of two was worse than two discrepancies of one.

Note that, unlike many other sports scheduling problems, travel was not a criterion to be considered in this problem. This is because teams always travel home between away matches and thus the total distance travelled is independent of the schedule.

The costs were added together into a single cost to be minimised. Given that the penalty costs, although set so as to reflect the approximate relative importance of the constraints, were to some 
extent arbitrary, the goal was not exact optimisation but the production of a solution which would satisfy the client.

\section{Solution approach - Simulated Annealing}

One way to model the problem formally could have been by means of 0-1 decision variables $\mathrm{x}_{\text {hayr }}$ where $h$ is the home team, $a$ the away team, $y$ the year and $r$ the round, to be set to 1 if team $h$ has a home match against team $a$ in year $y$, round $r$, or 0 otherwise. Thus the number of decision variables would be 12 (years) X 10 (home teams) X 10 (away teams) X 7 (rounds), i.e. 8400 . However, it was realised that the number of decision variables could be reduced significantly by taking advantage of the problem structure, as follows.

There are 360 matches to be scheduled. For each match $i(i=1, \ldots, 360)$, the home team $H_{i}$, the away team $A_{i}$ and the year $Y_{i}$ are known. What needs to be determined is $R_{i}$, the round in which the match is to be played. In a genuine solution, $R_{i}$ needs to be $1,2,3,4,4 a, 5$ or 6 , but in interim phases of the solution approach $R_{i}$ was also allowed to be 0 (unscheduled) or 7 (a dummy round), as explained below. Thus we have 360 decision variables, each of which may take one of nine values.

A solution for any year can be configured in a matrix as in Table 1, which shows the schedule for 2012. This structure is used in many papers - see, for example, di Gaspero and Schaerf (2007).

\begin{tabular}{|c|l|c|c|c|c|c|c|c|}
\hline \multicolumn{2}{|l|}{} & \multicolumn{9}{|c|}{ ROUND } \\
\hline Number & County & 1 & 2 & 3 & 4 & $4 a$ & 5 & 6 \\
\hline 1 & Bedfordshire & -5 & -10 & +8 & +4 & & -2 & +7 \\
\hline 2 & Buckinghamshire & -3 & +5 & -9 & +6 & & +1 & -8 \\
\hline 3 & Cambridgeshire & +2 & & +4 & +10 & -7 & -6 & -9 \\
\hline 4 & Cumberland & +9 & +6 & -3 & -1 & & -8 & +5 \\
\hline 5 & Hertfordshire & +1 & -2 & +10 & +8 & & -7 & -4 \\
\hline 6 & Lincolnshire & +8 & -4 & +7 & -2 & & +3 & -10 \\
\hline 7 & Norfolk & -10 & & -6 & +9 & +3 & +5 & -1 \\
\hline 8 & Northumberland & -6 & +9 & -1 & -5 & & +4 & +2 \\
\hline 9 & Staffordshire & -4 & -8 & +2 & -7 & & +10 & +3 \\
\hline 10 & Suffolk & +7 & +1 & -5 & -3 & & -9 & +6 \\
\hline
\end{tabular}

Table 1 - schedule matrix for 2012

Here the row shows the team and the column the round. A + sign signifies that the team played at home; a - sign signifies that the team played away.

Simulated Annealing (SA), a well-known metaheuristic technique, was then used to produce a good solution. It is not claimed that SA is necessarily the best possible method for solving this problem, but personal experience and that of many published researchers shows that it is a method that can produce very good solutions to combinatorial problems - see, for example, Suman \& Kumar (2006) for a survey of SA implementations. The main reason for using SA in this case rather than one of the many plausible alternatives was simple availability and speed; the work was being carried out pro bono and a satisfactory solution was more important than an exact optimum, even if agreement could have been reached as to what optimality actually meant for this problem.

The first stage of any SA implementation is to create an initial solution from which to proceed. In this case the initial solution was created by assigning all of the matches for the years 2012, 2013 and 2014 to their rounds but leaving all other matches unscheduled, i.e. with $R_{i}=0$. Since match absence is defined as a soft rather than a hard constraint, there is no need to include all matches in the initial 
solution; the size of the absence penalty is enough to ensure that all matches will be properly allocated in final solutions.

After the initial solution had been created, the matches for years 2015 to 2023 were randomly ordered and matches generating perturbations (see below) were considered in this order. When all matches had been considered, the matches were reordered randomly and the process continued until the total prespecified number of iterations (see below) had been reached.

\section{Perturbations}

Any SA implementation proceeds by perturbing a "current" solution to create new solutions which may or may not be accepted as the new current solution from which further perturbations are made. In many implementations such perturbations can be defined very simply. For example, Willis and Terrill (1994), in their system for scheduling cricket in Australia, used simple moves of one match from one date to another and swaps of dates between two matches as their perturbations. However, this would not have worked in this case.

The reason for this is that the structure of a solution is such that, once all matches have been scheduled, very few simple moves of a single match between rounds or swaps between rounds would result in a feasible schedule, since for the most part that would then imply at least one team playing two matches on the same date. Thus any solution would have had a very small feasible neighbourhood, severely inhibiting the success of any metaheuristic approach such as SA.

This problem was resolved partly by the addition of an extra dummy round (Round 7), for which the desired number of matches was zero, and partly by a complex definition of perturbations (and thus neighbourhoods), as detailed below. This approach was similar to that used by Wright (1994), for example, when scheduling professional cricket matches in England.

The perturbations generated by a single match $M_{i}$, with home team $H_{i}$ and away team $A_{i}$ and currently scheduled in Round R, are as listed below. All perturbations affect the schedule for only a single year, since the year for every match is predetermined. Perturbations are considered only if they do not result in any team having more than one match scheduled within the same Round in the same year, so as to preserve feasibility. Thus these perturbations are all special cases of "ejection chains", as described by Glover (1996).

1. Move $M_{i}$ from round $R$ (which could be zero, i.e. unscheduled) to any other Round $R^{*}$ for which neither team $H_{i}$ nor team $A_{i}$ has a match currently scheduled.

2. Move $M_{i}$ from round $R$ to any Round $R^{*}$ and move another match $M_{j}$ currently scheduled in Round $R^{*}$ and for which one of $H_{i}$ and $A_{i}$ is the same as one of $H_{j}$ and $A_{j}$ (i.e. matches $M_{i}$ and $M_{j}$ have a team in common) from Round $\mathrm{R}^{*}$ to any other Round $\mathrm{R}^{* *}$ (which could be the same as $\mathrm{R})$.

3. As 2 above, but with the addition of another move: a match $M_{k}$ currently scheduled in Round $R^{* *}$ and for which one of $H_{j}$ and $A_{j}$ is the same as one of $H_{k}$ and $A_{k}$ (i.e. matches $M_{j}$ and $M_{k}$ have a team in common) is moved from Round $\mathrm{R}^{* *}$ to any other Round $\mathrm{R}^{* * *}$ (which could be the same as either $\mathrm{R}$ or $\mathrm{R}^{*}$ ).

4. Again as 2 above, but with the addition of a different move: this time another match $M_{k}$ currently scheduled in Round $R^{*}$ and for which one of $H_{i}$ and $A_{i}$ is the same as one of $H_{k}$ and $A_{k}$ (i.e. matches $M_{i}$ and $M_{k}$ have a team in common) is moved from Round $R^{*}$ to any other Round $\mathrm{R}^{* * *}$ (which could be the same as either $\mathrm{R}$ or $\mathrm{R}^{* *}$ ). 
5. Move $M_{i}$ from round $R$ to any other Round $R^{*}$ and perform a Kempe Chain perturbation within these two rounds. In a Kempe Chain perturbation - see, for example, Morgernstern \& Shapiro (1989) - the initial move of $M_{i}$ from Round $R$ to Round $R^{*}$ displaces one or two matches from Round $R^{*}$, because they involve one of the same teams as $M_{i}$. These matches are then moved to Round $R$, but this in turn may displace other matches, which are moved to Round $R^{*}$, and so on, until feasibility is reached. Sometimes this may involve every single match being swapped between Rounds $\mathrm{R}$ and $\mathrm{R}^{*}$, but not always. Perturbations of Type 1 above are special cases of Kempe Chain moves, but most perturbations of Type 2 and all perturbations of Types 2, 3 and 4 are not, since they involve three or four rounds.

All perturbations of Types 1 to 5 above are tried in that order for the match $M_{i}$ until one is found which is accepted by the standard SA criterion, using parameters as detailed below. As soon as a perturbation is accepted, or if all possible perturbations have been tried for match $\mathrm{M}_{\mathrm{i}}$ and none has been accepted, the next match in the ordered list is used to generate further perturbations, and so on.

An exception to the above is that a tabu criterion is used: if a match has been moved in one of the two most recent perturbations to be accepted it is regarded as tabu, and it is not allowed to be involved in any further perturbation unless and until it ceases to be tabu (i.e. until two subsequent perturbations have been accepted). This criterion was added so as to avoid cycling between solutions; a tabu list length of 2 was found to be adequate for this purpose.

\section{Parameters}

As is usual for implementations of SA, a new solution was always accepted if the perturbation resulted in a decrease in overall cost, and otherwise accepted with a probability of $\mathrm{e}^{-(\Delta C / T)}$, where $\Delta C$ is the increase in overall cost and T is the "temperature", an entity which varies throughout the progress of the algorithm and whose value is controlled by parameters. There are several ways in which parameters can be defined and used for this purpose, as discussed by Dowsland \& Thompson (2012), and there is no clear evidence as to the best method, though it is clear that, whatever method is used, the values of the starting and ending temperatures are critical to the overall effectiveness of any SA implementation.

For this implementation, the simple geometric method was used, for which a starting temperature $T_{0}$, a number of iterations $\mathrm{N}$ and an ending temperature $\mathrm{T}_{\mathrm{N}}$ are specified. The temperature $\mathrm{T}$ starts at $\mathrm{T}_{0}$ and is subsequently multiplied by a constant parameter $\alpha$ at every iteration (i.e. every time a perturbation is considered) in order that the temperature reaches the ending temperature $T_{N}$ after $N$ iterations, at which point the algorithm stops and the best solution found is output and stored.

Some experimentation was used to find good values of $T_{0}$ and $T_{N}$, before they were finally set respectively as 50 and 0.5 . The number of perturbations in a run was set at 20 million; thus the factor $\alpha$ can be calculated to be approximately 0.99999977 .

As SA is a method involving a random element, every run will be different; to take advantage of this, the SA algorithm was run 500 times on a standard office PC (each run taking about five minutes).

\section{Results}

From the 500 runs, the mean cost was 2883, with a median of 2875 and a standard deviation of 208. The best solution had a cost of 2379 , and this was the solution selected for presentation to the client. The Match Absence, Stated Preference and Number of Matches costs were all zero, and there was also 
no special Home Round Spread Cost relating to Round 4a; this was very important since the client was regarding these as hard constraints.

The detailed costs for each county were as shown in Table 2.

\begin{tabular}{|l|c|c|c|c|}
\hline County & $\begin{array}{c}\text { Home/Away } \\
\text { Balance }\end{array}$ & Fixture spread & $\begin{array}{c}\text { Home Round } \\
\text { Spread }\end{array}$ & TOTAL \\
\hline Bedfordshire & 105 & 215 & 112 & 432 \\
\hline Buckinghamshire & 90 & 36 & 116 & 242 \\
\hline Cambridgeshire & 125 & 74 & 130 & 329 \\
\hline Cumberland & 45 & 54 & 78 & 177 \\
\hline Hertfordshire & 115 & 44 & 64 & 223 \\
\hline Lincolnshire & 85 & 39 & 78 & 202 \\
\hline Norfolk & 0 & 0 & 122 & 122 \\
\hline Northumberland & 145 & 68 & 126 & 339 \\
\hline Staffordshire & 35 & 33 & 44 & 112 \\
\hline Suffolk & 95 & 94 & 12 & 201 \\
\hline TOTAL & 840 & 657 & 882 & 2379 \\
\hline
\end{tabular}

$\underline{\text { Table } 2 \text { - costs of selected schedule }}$

These costs demonstrate some element of fairness in that no county was given a perfect schedule, but no county had a really disastrous one either.

It is not possible to determine the optimal solution and it does not appear at all straightforward to calculate meaningful bounds. However, it is of interest to compare these costs with the situation that would have arisen under the old system, whereby the schedule was simply repeated every three years. These costs are shown in Table 3.

\begin{tabular}{|l|c|c|c|c|c|}
\hline County & $\begin{array}{c}\text { Home/Away } \\
\text { Balance }\end{array}$ & Fixture spread & $\begin{array}{c}\text { Home Round } \\
\text { Spread }\end{array}$ & $\begin{array}{c}\text { Home } \\
\text { Preference }\end{array}$ & TOTAL \\
\hline Bedfordshire & 405 & 171 & 702 & 0 & 1278 \\
\hline Buckinghamshire & 30 & 342 & 702 & 0 & 1074 \\
\hline Cambridgeshire & 1005 & 171 & 702 & 0 & 1878 \\
\hline Cumberland & 270 & 171 & 702 & 0 & 1143 \\
\hline Hertfordshire & 180 & 0 & 702 & 0 & 882 \\
\hline Lincolnshire & 45 & 171 & 702 & 0 & 918 \\
\hline Norfolk & 0 & 0 & 6474 & 0 & 6474 \\
\hline Northumberland & 255 & 0 & 702 & 3000 & 3957 \\
\hline Staffordshire & 180 & 0 & 702 & 0 & 882 \\
\hline Suffolk & 300 & 171 & 702 & 0 & 1173 \\
\hline TOTAL & 2670 & 1197 & 12792 & 3000 & 19659 \\
\hline
\end{tabular}

Table 3 - costs of schedule under the old system

Note that this solution includes a Home Preference Cost for Northumberland, since its request for no more than one home match in the first three rounds would not have been met in 2016, 2017, 2019, 2020, 2022 and 2023. Also the Home Round Spread Cost relating to round 4a is here assigned to Norfolk, whereas it is the away teams (Bedfordshire, Buckinghamshire and Cambridgeshire) that feel the pain. 
Comparison between Tables 2 and 3 shows that, while there are a few individual costs lower in table 2 (e.g. the Fixture Spread Cost for Hertfordshire), overall every type of cost is much lower, and the total cost for every county is also much lower, in Table 2 than in Table 3.

The resulting nine-year schedule was welcomed by the MCCA and implemented for 2015 and beyond. To date no problems with the schedule have been reported.

\section{Reflections and conclusions}

Implementations of sports scheduling reported in the academic literature appear to be exclusively for a single season. While the choice of opponents for one season may be influenced by the choice of opponents in the previous season (see, for example, Wright (1992)), this does not usually affect scheduling. If a system is to be used for several seasons it is assumed that the general constraints and preferences for one season are not in any way influenced by the schedule produced for the preceding season, except perhaps in the case of individual specific preferences requested by one of the teams for example, they may wish for their final home match to be against an opponent different from that faced in their final home match of the previous season.

This case therefore breaks new ground as regards the academic literature, in that there is a deliberate attempt to make the schedule for one season different in many respects from previous schedules. While the "season" may perhaps in this case be regarded as lasting three years, being the double round robin period, the full twelve year period considered corresponds to four full seasons.

Perhaps it is the amateur nature of this competition that makes it different. For professional competitions it could be argued that the more "traditional" criteria (such as home balance and travel distance) are much more important, partly because players do not have to fit their cricket around fulltime jobs and partly because the experience of playing at different grounds may be less variable. However, in the case of amateur cricket, not only is cricket necessarily subservient to other commitments such as employment and family holidays, it may also be very important whether a particular ground known for its generous hospitality is visited while the strawberries are in season!

\section{References}

Capua, R. O., Martins, S. L., \& Ribeiro, C. C. (2012) Timetabling and field assignment for training youth football teams in amateur leagues. PATAT 2012, 352.

della Croce, F., Tadei, R., \& Asioli, P. S. (1999). Scheduling a round robin tennis tournament under courts and players availability constraints. Annals of Operations Research, 92, 349-361.

Dowsland, K. A., \& Thompson, J. M. (2012). Simulated annealing. In Handbook of natural computing (pp. 1623-1655). Springer Berlin Heidelberg.

di Gaspero, L., \& Schaerf, A. (2007). A composite-neighborhood tabu search approach to the traveling tournament problem. Journal of Heuristics, 13(2): 189-207.

Glover, F. (1996). Ejection chains, reference structures and alternating path methods for traveling salesman problems. Discrete Applied Mathematics 65(1): 223-253.

Goossens, D., \& Spieksma, F. (2011). Scheduling an amateur indoor football league. In Proceedings of the 3rd International Conference on Mathematics in Sport (pp. 44-49). Springer Verlag. 
Johnston, M., \& Wright, M. (2014). Prior analysis and scheduling of the 2011 Rugby Union ITM Cup in New Zealand. Journal of the Operational Research Society, 65(8), 1292-1300.

Kendall G., Knust S., Ribeiro C.C., \& Urrutia, S (2010). Scheduling in sports: an annotated bibliography. Computers and Operations Research 37(1): 1-19.

Kyngäs, J., Nurmi, K., Kyngäs, N., Lilley, G., Salter, T., \& Goossens, D. (2016). Scheduling the Australian Football League. Journal of the Operational Research Society, 1-10.

Lin, F., \& Yang, J. Y. (2010). Track and field sports meeting scheduling based on genetic algorithm. Journal of Jilin Institute of Chemical Technology, 88-90.

Meng, F. W., Chen, K. C., Lin, K. C., \& Chen, R. C. (2014). Scheduling Volleyball Games using Linear Programming and Genetic Algorithm. Information Technology Journal, 13(15), 2411.

Morgernstern, C., \& Shapiro, H. (1989). Chromatic number approximation using simulated annealing. Technical Report CS86-1, Department of Computer Science, University of New Mexico (1989)

Nurmi, K., Goossens, D., \& Kyngäs, J. (2014). Scheduling a triple round robin tournament with minitournaments for the Finnish national youth ice hockey league. Journal of the Operational Research Society, 65(11), 1770-1779.

Recalde, D., Torres, R., \& Vaca, P. (2013). Scheduling the professional Ecuadorian football league by integer programming. Computers \& Operations Research, 40(10), 2478-2484.

Saur, M. C., Starr, K., Husted, M., \& Newman, A. M. (2012). Scheduling Softball Series in the Rocky Mountain Athletic Conference. Interfaces, 42(3), 296-309.

Schönberger J., Mattfeld D.C. \& Kopfer H. (2004). Memetic algorithm timetabling for non-commercial sport leagues. European Journal of Operational Research 153(1), 102-116.

Suman, B., \& Kumar, P. (2006). A survey of simulated annealing as a tool for single and multiobjective optimization. Journal of the Operational Research Society, 57(10), 1143-1160.

Trick, M.A. (2011). Sports scheduling. In: van Hentenryck P and Milano M (eds). Hybrid Optimization. Springer: New York, pp 489-508.

Westphal, S. (2014). Scheduling the German basketball league. Interfaces, 44(5), 498-508.

Willis, R. J., \& Terrill, B. J. (1994). Scheduling the Australian state cricket season using simulated annealing. Journal of the Operational Research Society, 45(3), 276-280.

Wright, M.B. (1992). A fair allocation of county cricket opponents. Journal of the Operational Research Society 43(3): 195-201.

Wright, M. B. (1994). Timetabling county cricket fixtures using a form of tabu search. Journal of the Operational Research Society, 45(7), 758-770.

Wright, M. B. (2005). Scheduling fixtures for New Zealand cricket. IMA Journal of Management Mathematics, 16(2), 99-112.

Wright, M.B. (2009). Fifty years of OR in Sport. Journal of the Operational Research Society 60: S161S168. 
Wright, M. B. (2010). OR/MS applied to cricket. In the Wiley Encyclopedia of Operations Research and Management Science, eds. J.J.Cochran, L.A.Cox, P.Keskinocak, J.P.Kharoufeh \& J.C.Smith (Hoboken, New Jersey, USA). 
Appendix - the schedules for 2012 to 2023

\begin{tabular}{|c|c|c|c|c|c|}
\hline \multicolumn{6}{|l|}{ YEAR 2012} \\
\hline Round 1 & Cambs v Bucks & Cumbs v Staffs & Herts v Beds & Lincs v Nbld & Sfk v Nfk \\
\hline Round 2 & Bucks v Herts & Cumbs v Lincs & Nbld $v$ Staffs & Sfk v Beds & \\
\hline Round 3 & Beds v Nbld & Cambs v Cumbs & Herts v Sfk & Lincs v Nfk & Staffs v Bucks \\
\hline Round 4 & Beds v Cumbs & Bucks v Lincs & Cambs v Sfk & Herts v Nbld & Nfk v Staffs \\
\hline Round 4a & Nfk v Cambs & & & & \\
\hline Round 5 & Bucks v Beds & Lincs v Cambs & Nfk v Herts & Nbld v Cumbs & Staffs v Sfk \\
\hline Round 6 & Beds v Nfk & Cumbs v Herts & Nbld v Bucks & Staffs v Cambs & Sfk $v$ Lincs \\
\hline \multicolumn{6}{|l|}{ YEAR 2013} \\
\hline Round 1 & Beds v Bucks & Cambs v Nfk & Lincs v Sfk & Nbld $v$ Herts & Staffs v Cumbs \\
\hline Round 2 & Beds v Staffs & Bucks v Nbld & Cumbs v Nfk & Herts v Lincs & Sfk v Cambs \\
\hline Round 3 & Cambs v Herts & Lincs v Cumbs & Nbld v Beds & Sfk v Staffs & \\
\hline Round 4 & Beds v Herts & Bucks v Sfk & Cumbs v Cambs & Nfk v Nbld & Staffs $v$ Lincs \\
\hline Round 4a & Nfk v Bucks & & & & \\
\hline Round 5 & Bucks v Cumbs & Cambs v Beds & Herts v Staffs & Nfk $v$ Lincs & Sfk v Nbld \\
\hline Round 6 & Cumbs v Sfk & Herts v Bucks & Lincs v Beds & Nbld v Cambs & Staffs v Nfk \\
\hline \multicolumn{6}{|l|}{ YEAR 2014} \\
\hline Round 1 & Beds v Sfk & Cumbs v Bucks & Herts v Cambs & Lincs $v$ Staffs & Nbld v Nfk \\
\hline Round 2 & Bucks v Cambs & Nbld v Lincs & Staffs v Herts & Sfk v Cumbs & \\
\hline Round 3 & Cambs v Lincs & Cumbs v Beds & Herts v Nfk & Staffs $\vee \mathrm{Nbld}$ & Sfk v Bucks \\
\hline Round 4 & Beds v Cambs & Bucks v Staffs & Lincs v Herts & Nfk v Cumbs & Nbld v Sfk \\
\hline Round 4a & Nfk v Beds & & & & \\
\hline Round 5 & Cambs v Nbld & Herts v Cumbs & Lincs v Bucks & Nfk v Sfk & Staffs $v$ Beds \\
\hline Round 6 & Beds v Lincs & Bucks v Nfk & Cambs v Staffs & Cumbs v Nbld & Sfk v Herts \\
\hline \multicolumn{6}{|l|}{ YEAR 2015} \\
\hline Round 1 & Beds $v$ Nfk & Bucks $v$ Herts & Nbld v Cumbs & Staffs v Cambs & Sfk v Lincs \\
\hline Round 2 & Cambs v Bucks & Cumbs v Staffs & Herts v Beds & Lincs v Nbld & Sfk v Nfk \\
\hline Round 3 & Bucks v Beds & Cambs v Sfk & Cumbs v Lincs & Herts v Nbld & \\
\hline Round 4 & Beds v Cumbs & Lincs v Cambs & Nfk v Herts & Nbld v Bucks & Staffs v Sfk \\
\hline Round 4a & Nfk v Staffs & & & & \\
\hline Round 5 & Bucks v Lincs & Cumbs v Herts & Nfk v Cambs & Nbld v Staffs & Sfk v Beds \\
\hline Round 6 & Beds v Nbld & Cambs v Cumbs & Herts v Sfk & Lincs v Nfk & Staffs v Bucks \\
\hline \multicolumn{6}{|c|}{ YEAR 2016} \\
\hline Round 1 & Bucks v Nbld & Cambs v Nfk & Cumbs v Sfk & Herts v Staffs & Lincs v Beds \\
\hline Round 2 & Beds v Bucks & Cambs v Herts & Staffs v Cumbs & Sfk v Nbld & \\
\hline Round 3 & Bucks v Cumbs & Herts v Lincs & Nbld v Beds & Staffs v Nfk & Sfk v Cambs \\
\hline Round 4 & Beds v Staffs & Cumbs v Cambs & Lincs v Sfk & Nfk v Bucks & Nbld v Herts \\
\hline Round 4a & Nfk v Lincs & & & & \\
\hline Round 5 & Cambs v Beds & Herts v Bucks & Lincs v Cumbs & Nfk v Nbld & Sfk v Staffs \\
\hline Round 6 & Beds v Herts & Bucks v Sfk & Cumbs v Nfk & Nbld v Cambs & Staffs v Lincs \\
\hline
\end{tabular}


YEAR 2017

\begin{tabular}{|l|l|l|l|l|l|l|l}
\hline Round 1 & Cambs v Lincs & Cumbs v Beds & Herts v Nfk & Staffs v Nbld & Sfk v Bucks \\
\hline Round 2 & Beds v Cambs & Bucks v Nfk & Herts v Cumbs & Lincs v Staffs & Nbld v Sfk \\
\hline Round 3 & Cambs v Nbld & Lincs v Bucks & Staffs v Beds & Sfk v Herts & \\
\hline Round 4 & Beds v Lincs & Bucks v Staffs & Cumbs v Nbld & Herts v Cambs & Nfk v Sfk \\
\hline Round 4a & Nfk v Cumbs & & & \\
\hline Round 5 & Bucks v Cambs & Nfk v Beds & Nbld v Lincs & Staffs v Herts & Sfk v Cumbs \\
\hline Round 6 & Beds v Sfk & Cambs v Staffs & Cumbs v Bucks & Lincs v Herts & Nbld v Nfk \\
\hline
\end{tabular}

\section{YEAR 2018}

\begin{tabular}{|l|l|l|l|l|l|}
\hline Round 1 & Bucks v Beds & Cambs v Cumbs & Herts v Nbld & Lincs v Nfk & Staffs v Sfk \\
\hline Round 2 & Cumbs v Staffs & Lincs v Cambs & Nbld v Bucks & Sfk v Beds & \\
\hline Round 3 & Beds v Nbld & Bucks v Lincs & Cumbs v Herts & Staffs v Cambs & Sfk v Nfk \\
\hline Round 4 & Beds v Cumbs & Cambs v Bucks & Herts v Sfk & Lincs v Nbld & Nfk v Staffs \\
\hline Round 4a & Nfk v Herts & & & \\
\hline Round 5 & Herts v Beds & Nfk v Cambs & Nbld v Cumbs & Staffs v Bucks & Sfk v Lincs \\
\hline Round 6 & Beds v Nfk & Bucks v Herts & Cambs v Sfk & Cumbs v Lincs & Nbld v Staffs \\
\hline
\end{tabular}

\section{YEAR 2019}

\begin{tabular}{|l|l|l|l|l|l|l|}
\hline Round 1 & Cambs v Nfk & Herts v Bucks & Lincs v Cumbs & Nbld v Beds & Sfk v Staffs \\
\hline Round 2 & Beds v Herts & Bucks v Sfk & Cumbs v Cambs & Staffs v Lincs & \\
\hline Round 3 & Bucks v Nbld & Cambs v Beds & Cumbs v Nfk & Herts v Staffs & Lincs v Sfk \\
\hline Round 4 & Beds v Bucks & Nfk v Lincs & Nbld v Herts & Staffs v Cumbs & Sfk v Cambs \\
\hline Round 4a & Nfk v Nbld & & & \\
\hline Round 5 & Beds v Staffs & Cumbs v Sfk & Herts v Lincs & Nfk v Bucks & Nbld v Cambs \\
\hline Round 6 & Bucks v Cumbs & Cambs v Herts & Lincs v Beds & Staffs v Nfk & Sfk v Nbld \\
\hline
\end{tabular}

\section{YEAR 2020}

\begin{tabular}{|c|c|c|c|c|c|}
\hline Round 1 & Beds v Lincs & Bucks v Cambs & Nbld $v$ Nfk & Staffs v Herts & Sfk v Cumbs \\
\hline Round 2 & Beds v Sfk & Cambs v Nbld & Cumbs v Bucks & Herts v Nfk & Lincs v Staffs \\
\hline Round 3 & Cumbs v Beds & Herts v Cambs & Lincs v Bucks & Staffs v Nbld & \\
\hline Round 4 & Beds v Cambs & Bucks v Staffs & Nfk v Cumbs & Nbld v Lincs & Sfk v Herts \\
\hline Round 4a & Nfk v Sfk & & & & \\
\hline Round 5 & Cambs v Staffs & Cumbs v Nbld & Lincs v Herts & Nfk v Beds & Sfk v Bucks \\
\hline Round 6 & Bucks v Nfk & Cambs v Lincs & Herts v Cumbs & Nbld v Sfk & Staffs v Beds \\
\hline
\end{tabular}

\section{YEAR 2021}

\begin{tabular}{|l|l:l|l|l|l|}
\hline Round 1 & Bucks v Beds & Cumbs v Lincs & Herts v Nbld & Staffs v Cambs & Sfk v Nfk \\
\hline Round 2 & Beds v Cumbs & Cambs v Bucks & Herts v Sfk & Lincs v Nfk & Nbld v Staffs \\
\hline Round 3 & Bucks v Herts & Cumbs v Staffs & Lincs v Nbld & Sfk v Beds & \\
\hline Round 4 & Beds v Nbld & Cambs v Cumbs & Nfk v Herts & Staffs v Bucks & Sfk v Lincs \\
\hline Round 4a & Nfk v Cambs & & & \\
\hline Round 5 & Bucks v Lincs & Cambs v Sfk & Herts v Beds & Nfk v Staffs & Nbld v Cumbs \\
\hline Round 6 & Beds v Nfk & Cumbs v Herts & Lincs v Cambs & Nbld v Bucks & Staffs v Sfk \\
\hline & & & &
\end{tabular}




\begin{tabular}{|c|c|c|c|c|c|}
\hline Round 1 & Beds $v$ Herts & Bucks v Nbld & Cambs v Nfk & Lincs v Sfk & Staffs $\vee$ Cumbs \\
\hline Round 2 & Cumbs v Cambs & Herts v Lincs & Nbld v Beds & Sfk v Staffs & \\
\hline Round 3 & Bucks v Cumbs & Cambs v Herts & Lincs v Beds & Staffs v Nfk & Sfk v Nbld \\
\hline Round 4 & Beds v Staffs & Cumbs v Sfk & Herts v Bucks & Nfk v Lincs & Nbld v Cambs \\
\hline Round 4a & Nfk v Bucks & & & & \\
\hline Round 5 & Beds v Bucks & Herts v Staffs & Lincs v Cumbs & Nfk v Nbld & Sfk v Cambs \\
\hline Round 6 & Bucks v Sfk & Cambs v Beds & Cumbs v Nfk & Nbld v Herts & Staffs v Lincs \\
\hline \multicolumn{6}{|l|}{ YEAR 2023} \\
\hline Round 1 & Beds v Sfk & Cambs v Staffs & Herts v Cumbs & Lincs v Bucks & Nbld v Nfk \\
\hline Round 2 & Bucks v Nfk & Cambs v Lincs & Cumbs v Beds & Staffs v Nbld & Sfk v Herts \\
\hline Round 3 & Cumbs v Nbld & Herts v Cambs & Lincs v Staffs & Sfk v Bucks & \\
\hline Round 4 & Beds v Lincs & Bucks v Cambs & Nfk v Cumbs & Nbld v Sfk & Staffs v Herts \\
\hline Round 4a & Nfk v Beds & & & & \\
\hline Round 5 & Cambs v Nbld & Cumbs v Bucks & Lincs v Herts & Nfk v Sfk & Staffs v Beds \\
\hline Round 6 & Beds v Cambs & Bucks v Staffs & Herts v Nfk & Nbld v Lincs & Sfk v Cumbs \\
\hline
\end{tabular}

\section{Key:}

Beds $=$ Bedfordshire

Bucks $=$ Buckinghamshire

Cambs = Cambridgeshire

Cumbs = Cumberland

Herts $=$ Hertfordshire

Lincs $=$ Lincolnshire

Nbld $=$ Northumberland

$\mathrm{Nfk}=$ Norfolk

Sfk = Suffolk

Staffs $=$ Staffordshire 